\title{
Amplitude and Sign Adjustment for Peak-to-Average-Power Reduction
}

\author{
Masoud Sharif, Cedric Florens, Maryam Fazel, and Babak Hassibi
}

\begin{abstract}
In this letter, we propose a method to reduce the peak-to-mean-envelope-power ratio (PMEPR) of multicarrier signals by modifying the constellation. For $M$-ary phase-shift keying constellations, we minimize the maximum of the multicarrier signal over the sign and amplitude of each subcarrier. In order to find an efficient solution to the aforementioned nonconvex optimization problem, we present a suboptimal solution by first optimizing over the signs, and then optimizing over the amplitudes given the signs. We prove that the minimization of the maximum of a continuous multicarrier signal over the amplitude of each subcarrier can be written as a convex optimization problem with linear matrix inequality constraints. We also generalize the idea to other constellations such as 16-quadrature amplitude modulation. Simulation results show that by an average power increase of $0.21 \mathrm{~dB}$, and not sending information over the sign of each subcarrier, PMEPR can be decreased by $5.1 \mathrm{~dB}$ for a system with 128 subcarriers.
\end{abstract}

Index Terms-Convex optimization, orthogonal frequency-division multiplexing (OFDM), peak-to-average-power ratio, sign adjustment.

\section{INTRODUCTION}

$\mathbf{H}$ IGH peak-to-mean-envelope-power ratio (PMEPR) of multicarrier signals is one of the major obstacles in implementing orthogonal frequency-division multiplexing (OFDM), xDSL, and other broadband multicarrier systems. The occurrence of the large peaks in the signal seriously hampers the efficiency of the power amplifier.

Over the years, different schemes have been proposed for PMEPR reduction, such as coding, deliberate clipping, selective mapping (SLM), reserved carriers, and tone injection [2]-[7]. In all these schemes, there is always a tradeoff between PMEPR and other parameters in the systems, including coding rate, average power, signal distortion, and bandwidth. Methods like coding usually give a worst-case guarantee on the PMEPR. On the other hand, there are other methods, such as SLM, that improve the probability distribution of PMEPR, i.e., reduce the probability of encountering large PMEPR.

Recently, in [1] and [8], an algorithm was proposed to choose the sign of each subcarrier in order to reduce the PMEPR. In this letter, we generalize this idea and adjust the sign and amplitude of each subcarrier to further reduce the PMEPR. The price

Paper approved by A. K. Khandani, the Editor for Coding and Information Theory of the IEEE Communications Society. Manuscript received February 13, 2004; revised October 20, 2004. This work was supported in part by the National Science Foundation under Grant CCR-0133818, in part by the Office of Naval Research under Grant N00014-02-1-0578, and in part by Caltech's Lee Center for Advanced Networking. This paper was presented in part at the IEEE International Conference on Communications, Paris, France, June 2004.

The authors are with the Department of Electrical Engineering, California Institute of Technology, Pasadena, CA 91125 USA (e-mail: masoud@systems.caltech.edu; florens@systems.caltech.edu; hassibi@systems.caltech.edu; maryam@cds.caltech.edu).

Digital Object Identifier 10.1109/TCOMM.2005.852830 to adjust the amplitude of the subcarrier is a slight increase in the average power, while maintaining the same minimum distance for the constellation. Even though the optimization over the signs is not a convex optimization problem, we show that the amplitude optimization can be written as a convex optimization problem using the bounded real lemma [9]. This enables us to solve the problem using convex optimization techniques [12] and add more practical constraints to the problem, such as limiting the amplitude of each subcarrier in order to bound the peak to average in the frequency domain.

Our approach can be considered as a method to refine the constellation for PMEPR reductions and use the sign of each subcarrier to reduce the PMEPR. Other methods to shape the constellation have appeared in [7], [10], and [11] to reduce the maximum of the samples of the multicarrier signal. In [7], extending the number of constellation points is proposed. However, in [10] and [11], constellation points are allowed to move within some distortion constraints. In this letter, we consider a different constellation modification, and we further show that reducing the peak of the continuous multicarrier signal by optimizing the amplitude of the subcarriers is a convex optimization problem. In our approach, we first reduce the peak by optimizing over the signs of the multicarrier signal, which is not a convex problem.

Simulation results show that the PMEPR can be significantly reduced by using just $0.21 \mathrm{~dB}$ (i.e., 5\%) average power increase. More specifically, for a system with 128 subcarriers, and considering the peaks with probability less than $10^{-2}$ as negligible, PMEPR is reduced from 10.3 to 3.1, i.e., a 5.1-dB PMEPR improvement. The PMEPR improvement increases as the threshold probability becomes smaller.

The letter is organized as follows. Section II introduces our notations and the statement of the problem, and further, reviews the sign optimization algorithm. Section III deals with amplitude optimization and proves that it is a convex problem using the bounded real lemma. Simulations results are presented in Section IV, and Section V concludes the letter.

\section{Definitions AND PRoblem Statement}

In this letter, we consider a normalized multicarrier signal $s_{C}(\theta)$ that consists of $n$ subcarriers. More specifically

$$
s_{C}(\theta)=\sum_{i=1}^{n} c_{i} e^{j \theta i}
$$

where $C=\left(c_{1}, \ldots, c_{n}\right)$ is the modulating vector, $c_{i}$ is chosen from some constellations like $M$-ary phase-shift keying (MPSK) or 16-quadrature amplitude modulation (QAM), and $\theta$ denotes time. Clearly, if $c_{i}$ is chosen from a binary phase-shift keying (BPSK) constellation and it adds up coherently, $s_{C}(\theta)$ 
will have a large peak of order $n$. Therefore, it is of great practical interest to reduce the peak of $s_{C}(\theta)$, without excessively increasing the average power and introducing large peaks in the modulating codeword $C$.

As a measure for the fluctuation of the multicarrier signal, we may define the PMEPR of $C$ as

$$
\operatorname{PMEPR}(C)=\max _{0 \leq \theta \leq 2 \pi} \frac{\left|s_{C}(\theta)\right|^{2}}{E\left\{\sum_{i=1}^{n}\left|c_{i}\right|^{2}\right\}}
$$

where the denominator is the average power of $s_{C}(\theta)$. Thus, if $c_{i}$ is chosen independently from a constellation with average power of $P_{\mathrm{av}}$, then $E\left\{\sum_{i=1}^{n}\left|c_{i}\right|^{2}\right\}=n P_{\mathrm{av}}$.

In this letter, we consider the PMEPR reduction by adjusting the sign and amplitude of each subcarrier. This method is a more general version of the scheme that was recently proposed in [1] and [8]. Here, we first consider MPSK constellations, and we then generalize the idea to other constellations as well.

Here is the statement of the problem. For any given complex vector $C=\left(c_{1}, \ldots, c_{n}\right)$ where $c_{i}$ is chosen from any MPSK constellation, find the solution to the following optimization problem:

$$
\begin{array}{cc}
\underset{\epsilon, u}{\operatorname{minimize}} & \max _{0 \leq \theta \leq 2 \pi}\left|\sum_{i=1}^{n} \epsilon_{i}\left(1+u_{i}\right) c_{i} e^{j \theta i}\right| \\
\text { subject to } & \epsilon_{i} \in\{+1,-1\} \\
& 0 \leq u_{i} \leq u_{\max } \\
& \sum_{i=1}^{n}\left|c_{i}\right|^{2}\left(1+u_{i}\right)^{2} \leq(1+\eta) n P_{\mathrm{av}}
\end{array}
$$

where $\epsilon=\left(\epsilon_{1}, \ldots, \epsilon_{n}\right)$ and $u \in \mathbf{R}^{n}$ are the optimization variables, $u_{i}$ denotes the amplitude variations of the $i$ th subcarrier, and $\eta$ denotes the average power increase. In order to limit the variation of the new modulating vector $C_{\epsilon, u}=\left(\epsilon_{1}(1+\right.$ $\left.\left.u_{1}\right) c_{1}, \ldots, \epsilon_{n}\left(1+u_{n}\right) c_{n}\right)$, we further constrain $u_{i}$ to be less than $u_{\max }$. The last constraint also implies that the average power increase is controlled by the parameter $\eta$. Clearly, the bound on $u_{i}$ limits the dynamic range of the quantizer in the transmitter. On the other hand, $\eta$ limits the total variations of the constellation points, while the minimum distance between the constellation points is fixed.

In summary, the price for reducing the PMEPR with our scheme is a slight increase in the average power, $\eta$ or $10 \log (1+\eta) \mathrm{dB}$, and sending no information over the sign of each subcarrier. For instance, Fig. 1 shows the modified quaternary phase-shift keying (QPSK) constellation. For constellations other than MPSK, we let outer points in the constellation move such that the minimum distance of the constellation points does not change. This is shown for the 16-QAM constellation in Fig. 2.

It is also worth noting that the receiver is not required to know the vectors $\epsilon$ and $u$. Therefore, for the decoding, the receiver may ignore the sign of each subcarrier, as it does not convey any information. Furthermore, the receiver may use the same decision region for the decoding of the constellation points as for the case where $u=0$. This is due to the fact that constellation points are only allowed to move outward, as shown in Figs. 1 and 2 .

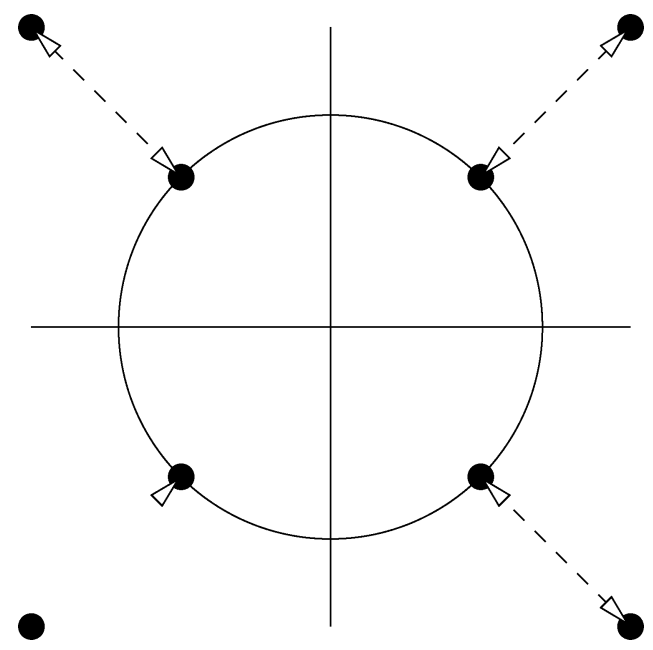

Fig. 1. Modified QPSK constellation.

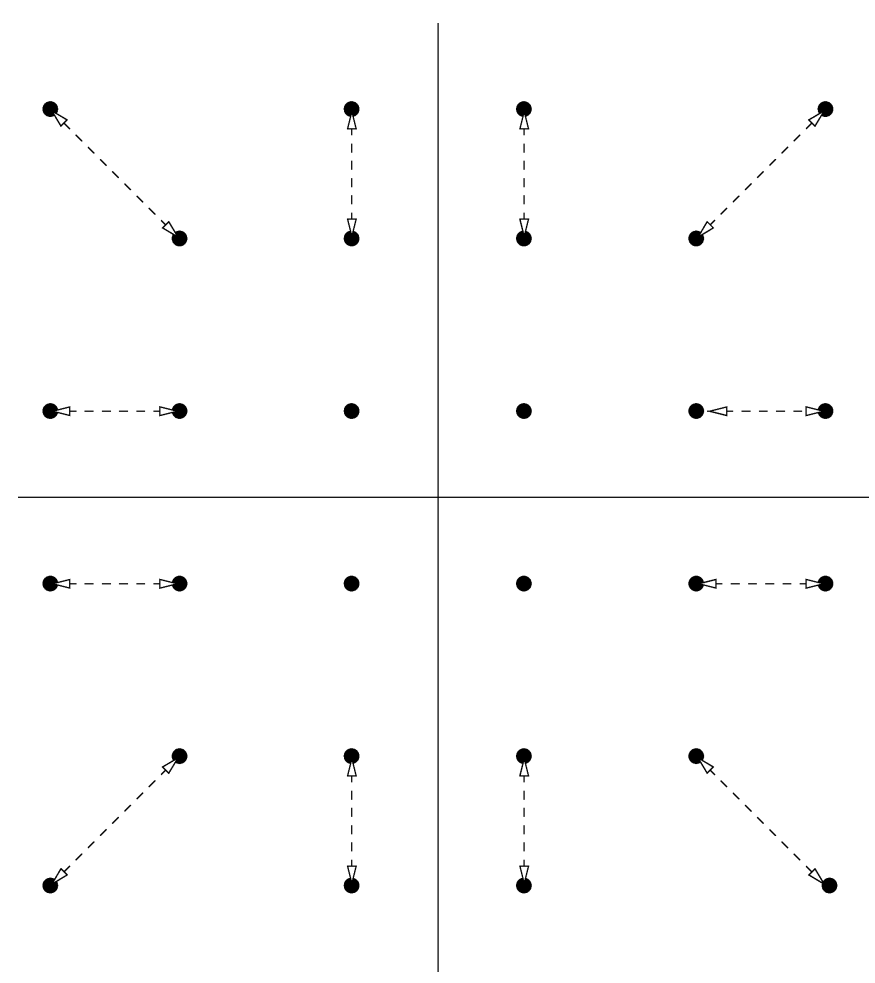

Fig. 2. Modified 16-QAM constellation.

In order to compensate the rate loss due to not sending information over the signs, the transmitter can double the constellation size at the expense of a $3-\mathrm{dB}$ average power increase (to preserve the minimum distance of the constellation). Therefore, the total cost for amplitude and sign adjustment will be $3+10 \log (1+\eta) \mathrm{dB}$ average power increase.

Clearly, the optimization problem, as stated in (3), is not convex, due to having an integer constraint, i.e., $\epsilon_{i} \in\{+1,-1\}$. However, assuming that $u_{i}=0$ for all $i$, we can find a suboptimal solution for the signs using the result of [1] and [8]. Afterwards, given $c_{i}$ and $\epsilon_{i}$, we show in Section IV that the optimization over $u_{i}$ is convex, and can be done very efficiently.

In what follows, we briefly review the sign optimization algorithm. 
Algorithm 1: For any $C=\left(c_{1}, \ldots, c_{n}\right)$, let $k$ be an integer greater than 1 and $\left|c_{i}\right| \leq \sqrt{E_{\max }}$. Then $\epsilon_{1}=1$, and $\epsilon_{j}$ is recursively determined as the minus sign of

$$
\sum_{p=1}^{2 k n} \sinh \left\{\alpha^{*} \sum_{r=1}^{j-1} \epsilon_{r} a_{p r}\right\} \sinh \left(\alpha^{*} a_{p j}\right) \prod_{r=j+1}^{n} \cosh \left\{\alpha^{*} a_{p r}\right\}
$$

for $j=2, \ldots, n$, where $\alpha^{*}=\sqrt{2 \log 4 k n / n E_{\max }}$

$$
a_{p i}= \begin{cases}\Re\left\{c_{i} e^{j \theta_{p} i}\right\}, & 1 \leq p \leq k n \\ \Im\left\{c_{i} e^{j \theta_{p} i}\right\}, & k n+1 \leq p \leq 2 k n\end{cases}
$$

and $\theta_{p}=2 \pi p / k n$.

It can be shown that the PMEPR of the codeword $C_{\epsilon}=$ $\left(\epsilon_{1} c_{1}, \ldots, \epsilon_{n} c_{n}\right)$ is less than $\gamma \log n$ for any $n$ where $\gamma$ is a constant independent of $n$. Even though Algorithm 1 does not give us the best signs, it is shown in [8] that it can significantly reduce the PMEPR. Now by further optimizing over the $u_{i}$, we can further reduce the PMEPR at the price of a slight increase in the average power. This gives us another degree of freedom to trade the PMEPR with a negligible average power increase, and without deteriorating the minimum distance of the constellation.

One might ask whether changing the order of the optimization might improve the PMEPR reduction. Intuitively, balancing the maximum of a multicarrier signal which is already fairly balanced by optimizing over the sign of each subcarrier requires less average power increase $\eta$ than the case where we first optimize the constellation over $u_{i}$. Simulation results also confirm this.

\section{AMPLitude AdJUSTMENT Using CONVEX OPtimizATION}

In this section, we solve the problem of minimizing the peak of the multicarrier signal over $u_{i}$ given the signs and the information symbols $c_{i}$, and we show that it is a convex problem with a linear matrix inequality (LMI) constraint [12]. We further present a relaxation of the problem that leads to an approximate solution with less complexity by minimizing the maximum of the samples of the multicarrier signal.

First of all, we notice the fact that

$$
\sum_{i=1}^{n} c_{i} \epsilon_{i}\left(1+u_{i}\right) z^{-i}=H(z I-F)^{-1} G
$$

where $H^{t}=\left[c_{1} \epsilon_{1}\left(1+u_{1}\right), \ldots, c_{n} \epsilon_{n}\left(1+u_{n}\right)\right], z=e^{j \theta}, G^{t}=$ $[1,0, \ldots, 0]$, and

$$
F=\left[\begin{array}{cccc}
0 & \ldots & 0 & \\
1 & 0 & \ldots & \\
\vdots & \vdots & & \\
0 & \ldots & 1 & 0
\end{array}\right]
$$

Given $\epsilon_{i}$ and $c_{i}$, we can then restate (3) as the following optimization problem:

$$
\begin{array}{ll}
\operatorname{minimize} & \gamma \\
\text { subject to } & \left\|H(z I-F)^{-1} G\right\|_{\infty} \leq \gamma \\
& 0 \leq u_{i} \leq u_{\max } \\
& \sum_{i=1}^{n}\left|c_{i}\right|^{2}\left(1+u_{i}\right)^{2} \leq(1+\eta) n P_{\mathrm{av}} .
\end{array}
$$

In order to show that the above problem is convex, we use the bounded real lemma [9].

Lemma 1 (Bounded Real Lemma): Suppose $\gamma>0$ and $F$ is stable. Then the following two statements are equivalent.

1) $\left\|H(z I-F)^{-1} G\right\|_{\infty}<\gamma$.

2) There exists a Hermitian $Y$ such that

$$
\mathcal{H}=\left[\begin{array}{ccc}
-Y+F^{*} Y F & H^{*} & F^{*} Y G \\
H & \gamma I & 0 \\
G^{*} Y F & 0 & \gamma I+G^{*} Y G
\end{array}\right]>0 .
$$

Since the matrix $\mathcal{H}$ is linear in the entries of the matrix $H$, we can see that the constraint in the optimization problem of (6) is an LMI [12].

Therefore, given the values of $\epsilon_{i}$ and $c_{i}$, we can state the minimization in (3) over $u_{i}$ as the following convex optimization problem:

$$
\begin{array}{ll}
\text { minimize } & \gamma \\
\text { subject to } & \mathcal{H} \geq 0 \\
& 0 \leq u_{i} \leq u_{\max } \\
& \sum_{i=1}^{n}\left|c_{i}\right|^{2}\left(1+u_{i}\right)^{2} \leq(1+\eta) n P_{\mathrm{av}}
\end{array}
$$

where $H^{t}=\left[c_{1} \epsilon_{1}\left(1+u_{1}\right), \ldots, c_{n} \epsilon_{n}\left(1+u_{n}\right)\right]$, and the matrix $Y$ in $\mathcal{H}$ is Hermitian.

This problem is a semidefinite program (SDP) and can be solved globally and efficiently using interior-point methods. Software packages exist that implement these methods; we use the recent package SeDuMi 1.02 [13].

Since the size of the LMI in the above SDP is relatively large, the computational load is still high for practical purposes. In fact, the complexity is $O\left(n^{6}\right)$, even though exploiting the structure of the LMI can lead to faster implementations [12]. Another way to lower the computational load with very little loss in accuracy is to discretize $s_{C}(\theta)$ and then solve the discretized problem. That is, instead of minimizing the maximum of $s_{C_{\epsilon, u}}(\theta)$ over $1 \leq \theta \leq 2 \pi$, we consider minimization of the maximum of $k n$ uniform samples of $s_{C_{\epsilon, u}}(\theta)$ at $\theta_{p}=2 \pi p / k n$ for $p=1, \ldots, k n .{ }^{1}$ This certainly has much less complexity, and can be written as a quadratically constrained quadratic program [12], which is solved much more efficiently than the original SDP. We use SeDuMi for solving this problem, as well. Furthermore, using the relationship between the maximum of $s_{C_{\epsilon, u}}(\theta)$ over $\theta$ and the maximum over $\theta_{i}$, we can make our approximation practically accurate by choosing $k=4$ [14], [15].

More specifically, this optimization problem can be written as

$$
\begin{array}{ll}
\text { minimize } & \gamma \\
\text { subject to } & 0 \leq u_{i} \leq u_{\max } \\
& \left|\Re\left\{s_{C}\left(\theta_{p}\right)\right\}\right|^{2}+\left|\Im\left\{s_{C}\left(\theta_{p}\right)\right\}\right|^{2} \leq \gamma, \\
& \text { for } p=1, \ldots, k n \\
& \sum_{i=1}^{n}\left|c_{i}\right|^{2}\left(1+u_{i}\right)^{2} \leq(1+\eta) n P_{\mathrm{av}} .
\end{array}
$$

${ }^{1}$ It is worth mentioning that the resulting $s_{C_{\epsilon, u}}\left(\theta_{p}\right)$ is the oversampled inverse fast Fourier transform of the vector $C_{\epsilon, u}$ 


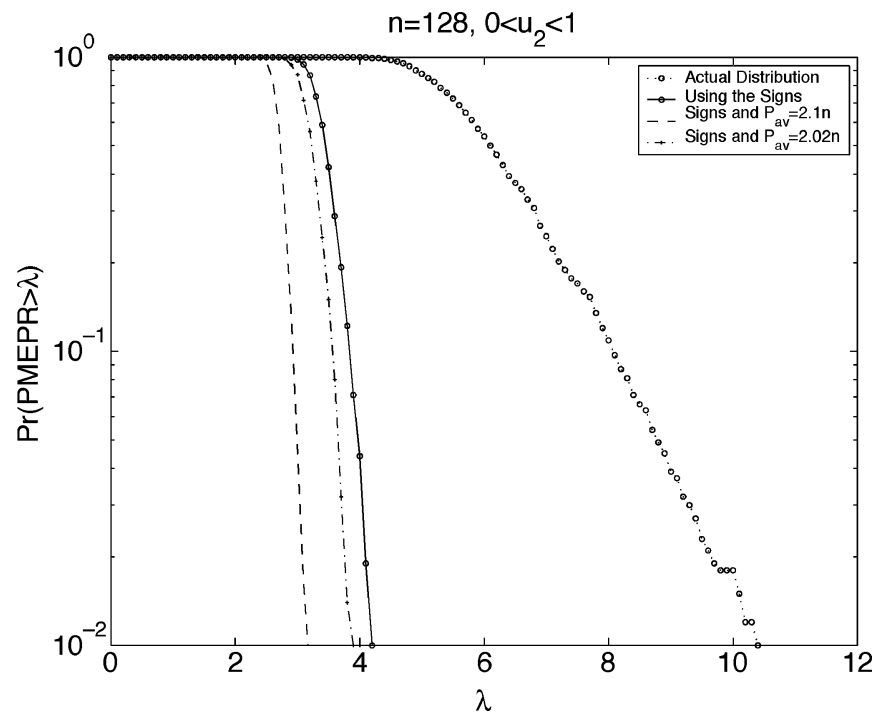

Fig. 3. CCDF of the PMEPR for QPSK by optimizing over $\epsilon_{i}$ and $u_{i}$ for $n=$ $128, u_{\max }=1$, and $\eta=0.01,0.05$.

Simulation results show that the solution to the problems in (7) and (8) are very close by choosing $k=4$. Therefore, in the simulations section, we solve the problem in (8) to optimize over $u_{i}$ instead of solving (7), which requires more computation.

\section{Simulation RESUlts}

As we discussed in the previous sections, there is a tradeoff between PMEPR reduction and average power increase $\eta$, and also the range of variation for $u_{i}$, i.e., $u_{\max }$. In this section, we carry out simulations to explore this tradeoff for $n=64$ and $n=128$, and for QPSK and 16-QAM constellations. The algorithm for designing the signs is applicable to any symmetric constellation. For the amplitude variation of the constellation points, we use the schemes shown in Figs. 1 and 2 for QPSK and 16-QAM, respectively.

Fig. 3 shows the complementary cumulative distribution function (CCDF) of PMEPR when $c_{i}$ is chosen from a QPSK constellation, and for different average power increases. Clearly, we need at least $0.21 \mathrm{~dB}(\eta=0.05)$ average power increase to get a noticeable PMEPR reduction after optimizing over the signs. As Fig. 3 suggests for $n=128$, the PMEPR can be decreased from 10.5 to 4.5 just by using the signs, and this can be further pushed down to 3.1 by also optimizing over $u_{i}$ with a little average power increase.

We can further do the simulations for a wider range of $u_{i}$, i.e., $u_{\max }=2$. As Fig. 4 shows for $n=128$, we can further reduce the PMEPR by allowing more degrees of freedom to each point; however, this causes large peak-to-average-power ratios for $c_{i}$, which is not practically favorable.

Fig. 5 also shows the PMEPR reduction when $c_{i}$ is chosen from a 16-QAM constellation, and the variation of the constellation points is as in Fig. 2. In summary, simulation results suggest that by expanding the constellation and increasing the average power by $0.21 \mathrm{~dB}$, the PMEPR of multicarrier signals can be decreased dramatically, i.e., from 10.5 to 3.1 for $n=128$.

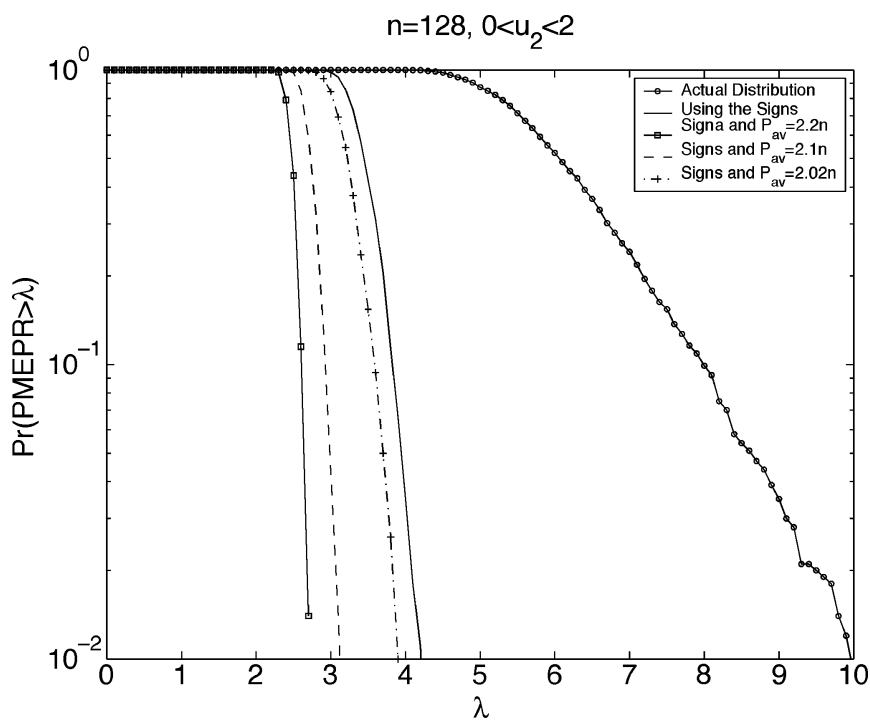

Fig. 4. CCDF of the PMEPR for QPSK by optimizing over $\epsilon_{i}$ and $u_{i}$ for $n=$ $128, u_{\max }=2$, and $\eta=0.01,0.05,0.1$.

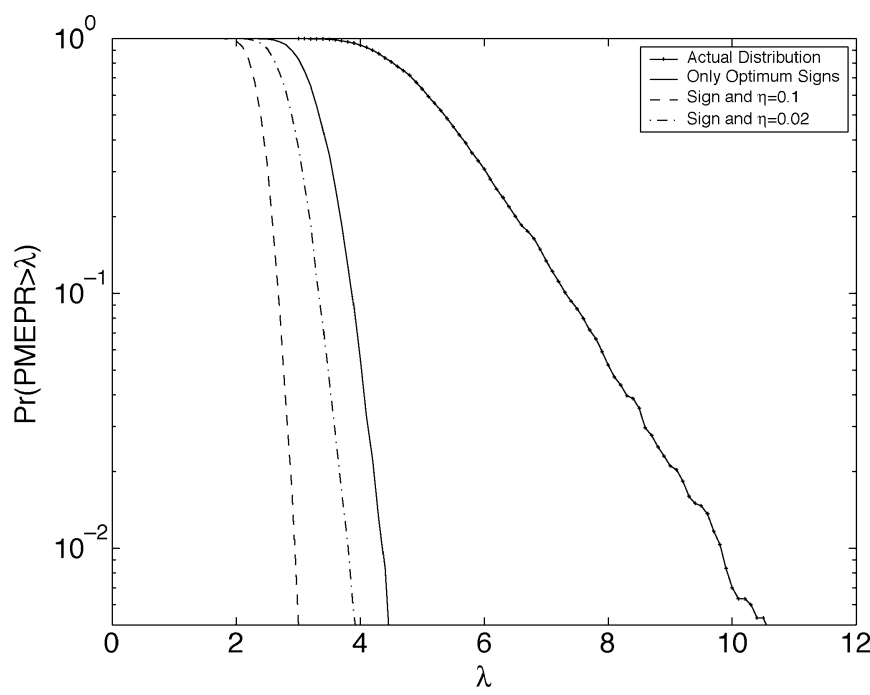

Fig. 5. CCDF of the PMEPR for 16-QAM constellation by optimizing over $\epsilon_{i}$ and $u_{i}$ for $n=64$ and $\eta=0.02,0.1$, and the constellation modification is according to Fig. 2 with $u_{\max }=0.3$ for outer points.

\section{CONCLUSION}

We presented a method to modify the constellation in order to reduce the PMEPR. This is done by minimizing the peak value of the multicarrier signal over the signs and amplitudes of the subcarriers with a slight increase in the average power. Since the problem is not a convex problem, we first used the algorithm in [1] to find a suboptimal solution for the signs, and then we used the convex optimization algorithm to optimize over the signs. Simulation results show significant improvement.

\section{REFERENCES}

[1] M. Sharif and B. Hassibi, "A deterministic algorithm that achieves PMEPR of $c \log n$ for multicarrier signals," in Proc. IEEE ICASSP, 2003, pp. 540-543.

[2] K. G. Paterson and V. Tarokh, "On the existence and construction of good codes with low peak to average power ratios," IEEE Trans. Inf. Theory, vol. 46, no. 9, pp. 1974-1986, Sep. 2000. 
[3] K. G. Paterson, "Generalized Reed-Muller codes and power control in OFDM modulation," IEEE Trans. Inf. Theory, vol. 46, no. 1, pp. 104-120, Jan. 2000.

[4] H. Ochiai and H. Imai, "Performance of the deliberate clipping with adaptive symbol selection for strictly bandlimited OFDM systems," IEEE J. Sel. Areas Commun., vol. 18, no. 11, pp. 2270-2277, Nov. 2000.

[5] X. Li and L. J. Cimini, "Effects of clipping and filtering on the performance of OFDM," in Proc. IEEE Veh. Technol. Conf., May 1997, pp. 1634-1638.

[6] S. H. Muller and J. B. Huber, "A comparison of peak power reduction schemes for OFDM," in Proc. IEEE Global Commun. Conf., 1997, pp. $1-5$.

[7] J. Tellado, Multicarrier Modulation with Low PAR: Applications to DSL and Wireless. Norwell, MA: Kluwer, 2000.

[8] M. Sharif and B. Hassibi, "Existence of codes with constant PMEPR and related designs," IEEE Trans. Signal Process., vol. 52, no. 10, pp. 2836-2847, Oct. 2004.

[9] B. Hassibi, A. H. Sayed, and T. Kailath, Indefinite-Quadratic Estimation and Control: A Unified Approach to $H^{2}$ and $H^{\infty}$ Theories. Philadelphia, PA: SIAM, 1999.
[10] D. Jones, "Peak power reduction in OFDM and DMT via active channel modification," in Proc. 32nd Asilomar Conf., Nov. 1999, pp. 1076-1079.

[11] A. Aggarwal and T. H. Meng, "Minimizing the peak to average power ratio of OFDM signals via convex optimization," in Proc. IEEE Globecom, Dec. 2003, pp. 2385-2389.

[12] S. Boyd and L. Vanderberghe, Convex Optimization. Cambridge, U.K.: Cambridge Univ. Press, 2003.

[13] J. F. Sturm, "Using SeDuMi 1.02, a MATLAB toolbox for optimization over symmetric cones," Optimization Methods and Software, pp. 625-653, 1999.

[14] M. Sharif, M. Gharavi-Alkhansari, and B. H. Khalaj, "On the peak to average power of OFDM signals based on oversampling," IEEE Trans. Commun., vol. 51, no. 1, pp. 72-78, Jan. 2003.

[15] G. Wunder and H. Boche, "Peak value estimation of bandlimited signals from their samples, noise enhancement, and a local characterization in the neighborhood of an extermum," IEEE Trans. Signal Process., vol. 51, no. 3, pp. 771-780, Mar. 2003. 